\title{
On some definitions and properties of generalized convex sets arising in the calculus of variations
}

\author{
Bernard DACOROGNA (bernard.dacorogna@epfl.ch) \\ Section de mathématiques, EPFL, 1015 Lausanne, Switzerland \\ Ana Margarida RIBEIRO (ana.ribeiro@epfl.ch) \\ Section de mathématiques, EPFL, 1015 Lausanne, Switzerland \\ Faculdade de Ciências e Tecnologia, UNL, Lisbon, Portugal
}

July 6, 2005

\begin{abstract}
We deal with generalized notions of convexity for sets. Namely, the polyconvexity, quasiconvexity, rank one convexity and separate convexity. The question has its origin in the calculus of variations. We try to systematize the results concerning these generalized notions imitating as much as possible the classical approach of convex analysis. Throughout the article, we will discuss the relations between the different convexities, separation and Carathéodory type theorems, the notion of hull of a set and extremal points.
\end{abstract}

\section{Introduction}

We discuss here the extension of the notion of convex set to generalized convex sets that are encountered in the vector valued calculus of variations and in partial differential equations. These are: polyconvex, quasiconvex and rank one convex set.

Contrary to classical convex analysis, where the notion of convex set precedes the one of convex function; this is not the case for the generalized ones. This is of course due to historical reasons. Morrey introduced the notions of polyconvex, quasiconvex and rank one convex functions in 1952 (although the terminology is the one of Ball). It was not until the systematic studies of partial differential equations and inclusions by Dacorogna-Marcellini and Müller-Šverák that the equivalent definitions for sets became an important issue. Moreover these notions were essentially seen through the different generalized convex hulls, leading somehow to terminologies that do not exactly covers the same concepts. One of the aims of the present paper is to try to imitate as much as possible 
the classical approach of convex analysis in the present context. This will, we hope, allow to clarify the situation.

In order to describe the content of our article, we have to get back to classical convex analysis. Here are important facts that we will try to mimic in the generalized context.

1) A set $E$ is convex if and only if its indicator function

$$
\chi_{E}(x)=\left\{\begin{array}{cc}
0 & \text { if } x \in E \\
+\infty & \text { if } x \notin E
\end{array}\right.
$$

is convex.

2) Important facts concerning convex sets are the separation and Carathéodory theorems.

3) The convex hull of a set $E$ is the smallest convex set, denoted co $E$, that contains $E$. As consequences of this definition, one finds that if

$$
\begin{aligned}
& \overline{\mathcal{F}}_{E}=\left\{f: \mathbb{R}^{m} \rightarrow \mathbb{R} \cup\{+\infty\}:\left.f\right|_{E} \leq 0\right\} \\
& \mathcal{F}_{E}=\left\{f: \mathbb{R}^{m} \rightarrow \mathbb{R}:\left.f\right|_{E} \leq 0\right\}
\end{aligned}
$$

then

$$
\begin{aligned}
& \operatorname{co} E=\left\{x \in \mathbb{R}^{m}: f(x) \leq 0, \text { for every convex } f \in \overline{\mathcal{F}}_{E}\right\} \\
& \overline{\operatorname{co} E}=\left\{x \in \mathbb{R}^{m}: f(x) \leq 0, \text { for every convex } f \in \mathcal{F}_{E}\right\}
\end{aligned}
$$

where $\overline{\operatorname{co} E}$ denotes the closure of co $E$.

4) Minkowski theorem for the convex hull of extreme points of compact sets.

The article is organized as follows.

In Section 3, we define the notions of polyconvex, quasiconvex and rank one convex set. The first and the third one are straightforward and are equivalent, as they should be, to the polyconvexity and rank one convexity of the indicator function. The second one is more delicate. Indeed one would have liked to define it as equivalent to the quasiconvexity of the indicator function; but quasiconvex functions allowed to take the value $+\infty$ are, at the moment, poorly understood. We will give a definition of quasiconvex set which is compatible with many of the desired properties that should have such definition. Notably we will have that

$$
E \text { convex } \Rightarrow E \text { polyconvex } \Rightarrow E \text { quasiconvex } \Rightarrow E \text { rank one convex }
$$

and all counterimplications turn out to be false whenever $N, n \geq 2$. This last result is better than the corresponding one for functions, since we have examples of rank one convex functions that are not quasiconvex (cf. Šverák [15]) only when $n \geq 2$ and $N \geq 3$.

Separation and Carathéodory type theorems exist for polyconvex sets and we will discuss these extensions in Section 4. 
In Section 5, we consider the definitions of polyconvex, quasiconvex and rank one convex hulls of a given set $E$ denoted respectively Pco $E$, Qco $E$, Rco $E$. They are, as they should be, the smallest polyconvex, quasiconvex and rank one convex set, respectively, that contains $E$. It turns out that for polyconvex sets (and in a similar way for rank one convex sets) we have

$$
\text { Pco } E=\left\{\xi \in \mathbb{R}^{N \times n}: f(\xi) \leq 0 \text {, for every polyconvex } f \in \overline{\mathcal{F}}_{E}\right\}
$$

as for the convex case. However, the representation of the closure of the hulls analogous to (2) is not true for general sets. We will discuss this question in details introducing three more types of hulls, namely

$$
\begin{gathered}
\operatorname{Pco}_{f} E=\left\{\xi \in \mathbb{R}^{N \times n}: f(\xi) \leq 0, \text { for every polyconvex } f \in \mathcal{F}_{E}\right\} \\
\mathrm{Qco}_{f} E=\left\{\xi \in \mathbb{R}^{N \times n}: f(\xi) \leq 0, \text { for every quasiconvex } f \in \mathcal{F}_{E}\right\} \\
\operatorname{Rco}_{f} E=\left\{\xi \in \mathbb{R}^{N \times n}: f(\xi) \leq 0, \text { for every rank one convex } f \in \mathcal{F}_{E}\right\} .
\end{gathered}
$$

It turns out that, in general,

$$
\overline{\mathrm{Pco} E} \underset{f}{\subset \mathrm{Pco}_{f} E}, \overline{\mathrm{Qco} E} \underset{\neq}{\subset} \mathrm{Qco}_{f} E \text { and } \overline{\mathrm{Rco} E} \underset{\neq}{\subset \mathrm{Rco}_{f} E .}
$$

However, if $E$ is compact, then

$$
\overline{\mathrm{Pco} E}=\mathrm{P}_{f} E .
$$

In Section 6 we will introduce the notion of extreme points in these generalized senses and establish Minkowski type theorems.

\section{Notations and preliminaries}

We recall the notation below (cf. Dacorogna [4]) used in the context of polyconvexity.

Notation 1 (i) For $\xi \in \mathbb{R}^{N \times n}$ we let

$$
T(\xi)=\left(\xi, \operatorname{adj}_{2} \xi, \ldots, \operatorname{adj}_{N \wedge n} \xi\right) \in \mathbb{R}^{\tau(N, n)}
$$

where $\operatorname{adj}_{s} \xi$ stands for the matrix of all $s \times s$ subdeterminants of the matrix $\xi$, $1 \leq s \leq N \wedge n=\min \{N, n\}$ and where

$$
\tau=\tau(N, n)=\sum_{s=1}^{m \wedge n}\left(\begin{array}{c}
N \\
s
\end{array}\right)\left(\begin{array}{l}
n \\
s
\end{array}\right) \text { and }\left(\begin{array}{c}
N \\
s
\end{array}\right)=\frac{N !}{s !(N-s) !} .
$$

In particular if $N=n=2$, then $T(\xi)=(\xi$, $\operatorname{det} \xi)$.

(ii) For $s \in \mathbb{N}$, let

$$
\Lambda_{s}=\left\{\lambda=\left(\lambda_{1}, \ldots, \lambda_{s}\right): \lambda_{i} \geq 0, \sum_{i=1}^{s} \lambda_{i}=1\right\} .
$$


We also introduce a useful notation when defining a quasiconvex set (cf. Definition 6).

Notation 2 Let $\Omega$ be the hypercube $(0,1)^{n}$ of $\mathbb{R}^{n}$. For an orthogonal transformation $R \in O(n)$,

- $W_{\text {per }}^{1, \infty}\left(R \Omega ; \mathbb{R}^{N}\right)$ will denote the space of periodic functions in $W^{1, \infty}\left(R \Omega ; \mathbb{R}^{N}\right)$, i.e. functions u verifying $u(R x)=u\left(R\left(x+e_{i}\right)\right)$ for all vectors $e_{i}$ of the canonical basis of $\mathbb{R}^{n}$ and all $x \in \Omega$;

- $\operatorname{Aff}_{\text {piec }}\left(R \Omega ; \mathbb{R}^{N}\right)$ will denote the space of piecewise affine functions in $R \Omega$;

- $\mathcal{W}_{R}$ will denote the space $W_{\text {per }}^{1, \infty}\left(R \Omega ; \mathbb{R}^{N}\right) \cap \operatorname{Aff}_{\text {piec }}\left(R \Omega ; \mathbb{R}^{N}\right)$ of functions whose gradients take only a finite number of values.

We now recall the different notions of convexity for functions.

Definition 3 (i) A function $f: \mathbb{R}^{m} \rightarrow \mathbb{R} \cup\{+\infty\}$ is said to be convex if

$$
f(\lambda \xi+(1-\lambda) \eta) \leq \lambda f(\xi)+(1-\lambda) f(\eta)
$$

for every $\lambda \in[0,1]$ and every $\xi, \eta \in \mathbb{R}^{m}$.

(ii) A function $f: \mathbb{R}^{N \times n} \rightarrow \mathbb{R} \cup\{+\infty\}$ is said to be polyconvex if there exists a convex function $g: \mathbb{R}^{\tau(N, n)} \longrightarrow \mathbb{R} \cup\{+\infty\}$ such that

$$
f(\xi)=g(T(\xi)) \text {. }
$$

(iii) A Borel measurable function $f: \mathbb{R}^{N \times n} \rightarrow \mathbb{R}$ is said to be quasiconvex if

$$
f(\xi) \operatorname{meas}(U) \leq \int_{U} f(\xi+D \varphi(x)) d x
$$

for every bounded open set $U \subset \mathbb{R}^{n}, \xi \in \mathbb{R}^{N \times n}$ and $\varphi \in W_{0}^{1, \infty}\left(U ; \mathbb{R}^{N}\right)$.

(iv) A function $f: \mathbb{R}^{N \times n} \rightarrow \mathbb{R} \cup\{+\infty\}$ is said to be rank one convex if

$$
f(\lambda \xi+(1-\lambda) \eta) \leq \lambda f(\xi)+(1-\lambda) f(\eta)
$$

for every $\lambda \in[0,1]$ and every $\xi, \eta \in \mathbb{R}^{N \times n}$ with $\operatorname{rank}(\xi-\eta)=1$.

(v) A function $f: \mathbb{R}^{m} \rightarrow \mathbb{R} \cup\{+\infty\}$ is said to be separately convex if

$$
f(\lambda \xi+(1-\lambda) \eta) \leq \lambda f(\xi)+(1-\lambda) f(\eta)
$$

for every $\lambda \in[0,1]$ and every $\xi, \eta \in \mathbb{R}^{m}$ with $\xi-\eta=s e_{i}$, for some $s \in \mathbb{R}$ and $i \in\{1, \ldots, m\}$ ( $e_{i}$ denoting the $i^{\text {th }}$-vector of the canonical basis of $\left.\mathbb{R}^{m}\right)$.

(vi) A Borel measurable function $f: \mathbb{R}^{N \times n} \rightarrow \mathbb{R}$ is said to be quasiaffine if both $f$ and $-f$ are quasiconvex. 
Remark 4 A good definition of quasiconvex functions equivalent to the weak lower semicontinuity of the corresponding integral taking the value $+\infty$ is not available at the moment. Moreover, if we allow it in the above definition, then the known implication

$$
f \text { quasiconvex } \Rightarrow f \text { rank one convex }
$$

is no longer true.

Equivalent conditions for polyconvexity and quasiconvexity are given in the next result. For the proofs see, respectively, Dacorogna [4, page 106] and Šverák [15].

Theorem 5 (i) A function $f: \mathbb{R}^{N \times n} \rightarrow \mathbb{R} \cup\{+\infty\}$ is polyconvex if and only if

$$
f\left(\sum_{i=1}^{\tau+1} \lambda_{i} \xi_{i}\right) \leq \sum_{i=1}^{\tau+1} \lambda_{i} f\left(\xi_{i}\right)
$$

whenever $\left(\lambda_{1}, \ldots, \lambda_{\tau+1}\right) \in \Lambda_{\tau+1}$ and

$$
T\left(\sum_{i=1}^{\tau+1} \lambda_{i} \xi_{i}\right)=\sum_{i=1}^{\tau+1} \lambda_{i} T\left(\xi_{i}\right)
$$
if

(ii) A Borel measurable function $f: \mathbb{R}^{N \times n} \rightarrow \mathbb{R}$ is quasiconvex if and only

$$
f(\xi) \leq \int_{R \Omega} f(\xi+D \varphi(x)) d x
$$

for $\Omega:=(0,1)^{n}$ and every $R \in O(n), \varphi \in W_{\text {per }}^{1, \infty}\left(R \Omega ; \mathbb{R}^{N}\right)$ and $\xi \in \mathbb{R}^{N \times n}$.

The different envelopes are then defined as

$$
\begin{aligned}
C f & =\sup \{g \leq f: g \text { convex }\} \\
P f & =\sup \{g \leq f: g \text { polyconvex }\}, \\
Q f & =\sup \{g \leq f: g \text { quasiconvex }\}, \\
R f & =\sup \{g \leq f: g \text { rank one convex }\} \\
S f & =\sup \{g \leq f: g \text { separately convex }\} .
\end{aligned}
$$

As well known we have that, provided $f: \mathbb{R}^{N \times n} \longrightarrow \mathbb{R}$, the following implications hold

$$
\begin{aligned}
f \text { convex } \Rightarrow f \text { polyconvex } & \Rightarrow f \text { quasiconvex } \\
\Rightarrow f \text { rank one convex } & \Rightarrow f \text { separately convex }
\end{aligned}
$$

and thus

$$
C f \leq P f \leq Q f \leq R f \leq S f \leq f .
$$




\section{Generalized notions of convexity}

We start giving the generalized definitions of convexity for sets.

Definition 6 (i) We say that $E \subset \mathbb{R}^{m}$ is convex if for every $\lambda \in[0,1]$ and $\xi, \eta \in E$, then

$$
\lambda \xi+(1-\lambda) \eta \in E
$$

(ii) We say that $E \subset \mathbb{R}^{N \times n}$ is polyconvex if there exists a convex set $K \subset$ $\mathbb{R}^{\tau(N, n)}$ such that

$$
\pi\left(K \cap T\left(\mathbb{R}^{N \times n}\right)\right)=E,
$$

where $\pi$ denotes the orthogonal projection of (the first component of) $\mathbb{R}^{\tau(N, n)}$ in $\mathbb{R}^{N \times n}$. Equivalently, $E$ is polyconvex if there exists a convex set $K \subset \mathbb{R}^{\tau(N, n)}$ such that

$$
\left\{\xi \in \mathbb{R}^{N \times n}: T(\xi) \in K\right\}=E .
$$

(iii) We say that $E \subset \mathbb{R}^{N \times n}$ is quasiconvex if we have

$$
\left.\begin{array}{c}
\xi+D \varphi(x) \in E, \text { a.e. } x \in R \Omega, \\
\text { for some } R \in O(n) \text { and } \varphi \in \mathcal{W}_{R}
\end{array}\right\} \Rightarrow \xi \in E
$$

$\left(\Omega\right.$ denoting the hypercube $\left.(0,1)^{n}\right)$.

(iv) Let $E \subset \mathbb{R}^{N \times n}$. We say that $E$ is rank one convex if for every $\lambda \in[0,1]$ and $\xi, \eta \in E$ such that $\operatorname{rank}(\xi-\eta)=1$, then

$$
\lambda \xi+(1-\lambda) \eta \in E .
$$

(v) We say that $E \subset \mathbb{R}^{m}$ is separately convex if for every $\lambda \in[0,1]$ and $\xi, \eta \in E$ such that $\xi-\eta=s e_{i}$, for some $s \in \mathbb{R}$ and $i \in\{1, \ldots, m\}$ ( $e_{i}$ denoting the $i^{\text {th }}$-vector of the canonical basis of $\mathbb{R}^{m}$ ), then

$$
\lambda \xi+(1-\lambda) \eta \in E .
$$

Remark 7 (i) The operator $\pi$ introduced in the above definition is more precisely defined as follows. If

$$
X=\left(X_{1}, \ldots, X_{\tau(N, n)}\right) \text { then } \pi(X)=\left(X_{1}, \ldots, X_{N \times n}\right) .
$$

In particular, if $N=n=2$ and $X=(\xi, \delta) \in \mathbb{R}^{2 \times 2} \times \mathbb{R}$, then $\pi(X)=\xi$.

(ii) The definitions of convex, rank one convex and separately convex sets are standard.

(iii) In what concerns polyconvexity, the more usual way to define it is with the condition in Theorem 8 below. However, the two conditions turn out to be equivalent. With our definition we get some coherence with the analogous notion for functions. 
We note that one could think, in view of Definition $3(i i)$, that a set $E$ is polyconvex if $T(E)$ is convex. This is however not true. Consider, for example, the polyconvex set $E=\{I, \xi\}$, where $I$ is the identity matrix and $\xi=\operatorname{diag}(2,0)$. Then $T(E)=\{(I, 1),(\xi, 0)\}$ which is not convex.

(iv) The best definition for quasiconvex sets is unclear. Several definitions have already been considered (see Dacorogna-Marcellini [5], Müller [11], Zhang [18]). The one we propose here is consistent with known properties for functions and have most properties which are desirable (cf. Theorem 11 below).

We first give an equivalent condition for polyconvexity.

Theorem 8 Let $E \subset \mathbb{R}^{N \times n}$. The following conditions are equivalent.

(i) $E$ is polyconvex.

(ii)

$$
\left.\begin{array}{c}
\sum_{i=1}^{I} \lambda_{i} T\left(\xi_{i}\right)=T\left(\sum_{i=1}^{I} \lambda_{i} \xi_{i}\right) \\
\xi_{i} \in E,\left(\lambda_{1}, \ldots, \lambda_{I}\right) \in \Lambda_{I}
\end{array}\right\} \Rightarrow \sum_{i=1}^{I} \lambda_{i} \xi_{i} \in E .
$$

Moreover one can take $I=\tau(N, n)+1$.

(iii) Denoting by $\operatorname{co} T(E)$ the convex hull of $T(E)$,

$$
E=\pi\left(\operatorname{co} T(E) \cap T\left(\mathbb{R}^{N \times n}\right)\right)
$$

or equivalently

$$
E=\left\{\xi \in \mathbb{R}^{N \times n}: T(\xi) \in \operatorname{co} T(E)\right\} .
$$

Proof. $(i) \Rightarrow(i i)$. Suppose

$$
\sum_{i=1}^{I} \lambda_{i} T\left(\xi_{i}\right)=T\left(\sum_{i=1}^{I} \lambda_{i} \xi_{i}\right),
$$

for some $\xi_{i} \in E$ and $\left(\lambda_{1}, \ldots, \lambda_{I}\right) \in \Lambda_{I}$. By hypothesis, $\xi_{i} \in \pi\left(K \cap T\left(\mathbb{R}^{N \times n}\right)\right)$ for some convex set $K \subset \mathbb{R}^{\tau(N, n)}$ and so $T\left(\xi_{i}\right) \in K$. Therefore $\sum_{i=1}^{I} \lambda_{i} T\left(\xi_{i}\right) \in$ co $K=K$ and, by (3), we conclude that $\sum_{i=1}^{I} \lambda_{i} \xi_{i} \in E$.

The fact that we can take $I=\tau(N, n)+1$ in $(i i)$ is a consequence of Carathéodory theorem (see Dacorogna [4, Theorem 1.3, page 106]).

$($ ii $) \Rightarrow($ iii $)$. We have to see that $E=\pi\left(\operatorname{co} T(E) \cap T\left(\mathbb{R}^{N \times n}\right)\right)$. Evidently $E$ is contained in the set in the right hand side. For the reverse inclusion, consider $\xi \in \pi\left(\operatorname{co} T(E) \cap T\left(\mathbb{R}^{N \times n}\right)\right)$. So, $T(\xi) \in \operatorname{co} T(E)$ and we can write

$$
T(\xi)=\sum_{i=1}^{I} \lambda_{i} T\left(\xi_{i}\right)
$$

for some $\xi_{i} \in E$ and $\left(\lambda_{1}, \ldots, \lambda_{I}\right) \in \Lambda_{I}$. We then use (ii) to get that $\xi \in E$, as wished. 
(iii) $\Rightarrow($ i $)$ This is immediate.

The next result shows the relation between the notions of convexity for sets and the corresponding notions for functions (the proof is straightforward).

Proposition 9 Let $E \subset \mathbb{R}^{N \times n}$ and $\chi_{E}$ denote the indicator function of $E$ :

$$
\chi_{E}(\xi)=\left\{\begin{array}{cc}
0 & \text { if } \xi \in E \\
+\infty & \text { if } \xi \notin E .
\end{array}\right.
$$

Then $E$ is, respectively, convex, polyconvex, rank one convex or separately convex, if and only if $\chi_{E}$ is, respectively, convex, polyconvex, rank one convex or separately convex.

Remark 10 One would have liked to have the same result for quasiconvex sets but, as already discussed, quasiconvex functions taking the value $+\infty$ are not considered here.

The convexity conditions are related in the following way.

Theorem 11 Let $E \subset \mathbb{R}^{N \times n}$. We have the following implications

$E$ convex $\Rightarrow E$ polyconvex $\Rightarrow E$ quasiconvex

$\Rightarrow E$ rank one convex $\Rightarrow E$ separately convex.

All counterimplications are false, as soon as $N, n \geq 2$.

Remark 12 We will see (cf. Proposition 28) that, as for the convex case: $E$, respectively, polyconvex, quasiconvex, rank one convex or separately convex implies that int $E$ is also, respectively, polyconvex, quasiconvex, rank one convex or separately convex. However, this is not anymore true for $\bar{E}$. Indeed we will give (cf. Proposition 28) an example of a bounded polyconvex set $E \subset \mathbb{R}^{2 \times 2}$ with $\bar{E}$ not even separately convex.

Proof. Part 1. We only prove the implications related to the notion of quasiconvexity since the others are trivial and well known.

(i) We prove that if $E$ is polyconvex then $E$ is quasiconvex. Assume that

$$
\xi+D \varphi(x) \in E, \text { a.e. } x \in R \Omega
$$

for some $R \in O(n)$ and $\varphi \in \mathcal{W}_{R}$. We can write $D \varphi(x) \in\left\{\eta_{1}, \ldots, \eta_{k}\right\}$, a.e. $x \in$ $R \Omega$ for some $\eta_{i}$ such that $\xi+\eta_{i} \in E, i=1, \ldots, k$. Defining

$$
\lambda_{i}=\operatorname{meas}\left\{x \in R \Omega: D \varphi(x)=\eta_{i}\right\},
$$

we have $\lambda_{i} \geq 0, \sum_{i=1}^{k} \lambda_{i}=1$. Since $\varphi$ is periodic and the functions $\operatorname{adj}_{s}$ are quasiaffine $(s=1, \ldots, N \wedge n)$ we have

$$
T(\xi)=\int_{R \Omega} T(\xi+D \varphi(x)) d x=\sum_{i=1}^{k} \lambda_{i} T\left(\xi+\eta_{i}\right) .
$$


Using the polyconvexity of the set $E$ we obtain that $\xi \in E$.

(ii) We now prove that if a set $E$ is quasiconvex then it is rank one convex. Let $\xi, \eta \in E$ be such that $\operatorname{rank}(\xi-\eta)=1$ and $\lambda \in(0,1)$. We will prove that $\lambda \xi+(1-\lambda) \eta \in E$. To achieve this, it is enough to find $R \in O(n)$ and $\varphi \in \mathcal{W}_{R}$ such that

$$
\lambda \xi+(1-\lambda) \eta+D \varphi(x) \in\{\xi, \eta\}, \text { a.e. } x \in R \Omega
$$

or equivalently

$$
D \varphi(x) \in\{(1-\lambda)(\xi-\eta),-\lambda(\xi-\eta)\}, \text { a.e. } x \in R \Omega .
$$

The result will then follows from the quasiconvexity of $E$. The construction of such $\varphi$ is standard for relaxation theorems (see, for example, Dacorogna [4]). We just outline the proof. Since $\operatorname{rank}(\xi-\eta)=1$, we can write $\xi-\eta=a \otimes \nu$ with $a \in \mathbb{R}^{N}$ and $\nu$ a unit vector in $\mathbb{R}^{n}$. Choose $R \in O(n)$ any orthogonal transformation such that $\operatorname{Re}_{1}=\nu$ ( $e_{1}$ denoting the first vector of the canonical basis) and define the function $h: \mathbb{R} \longrightarrow \mathbb{R}$ by

$$
h(s)=\left\{\begin{array}{l}
s, 0 \leq s \leq \lambda \\
\lambda, \lambda \leq s \leq 1
\end{array}\right.
$$

and $h(s+1)=h(s)+\lambda, \forall s \in \mathbb{R}$. Then $\varphi(x)=-\lambda(\xi-\eta) x+a h(\langle x ; \nu\rangle)$ satisfies the required conditions, which finishes the proof.

Part 2. We will next see that the reverse implications are, in general, not true. (i) There are polyconvex sets which are not convex. Consider, for example, the set $E=\{\xi, \eta\} \subset \mathbb{R}^{2 \times 2}$, where $\xi=\operatorname{diag}(1,0)$ and $\eta=\operatorname{diag}(0,1)$.

(ii) Quasiconvexity does not imply polyconvexity. Consider the matrices (cf. Dacorogna [4])

$$
\xi_{1}=\left(\begin{array}{ll}
1 & 0 \\
2 & 0
\end{array}\right), \quad \xi_{2}=\left(\begin{array}{ll}
0 & 1 \\
0 & 1
\end{array}\right), \quad \xi_{3}=\left(\begin{array}{cc}
-1 & -1 \\
0 & 0
\end{array}\right)
$$

and

$$
\eta=\left(\begin{array}{cc}
0 & 0 \\
2 / 3 & 1 / 3
\end{array}\right)
$$

We have

$$
T(\eta)=\frac{1}{3} T\left(\xi_{1}\right)+\frac{1}{3} T\left(\xi_{2}\right)+\frac{1}{3} T\left(\xi_{3}\right) .
$$

The set $E=\left\{\xi_{1}, \xi_{2}, \xi_{3}\right\}$ is not a polyconvex set since $\eta \notin E$. However, it is quasiconvex. Suppose $\xi+D \varphi \in E$ for some $\varphi \in \mathcal{W}_{R}$ where $R \in O(2)$. Since $\operatorname{rank}\left(\xi_{i}-\xi_{j}\right)=2$ for $i \neq j$, we have that the solution of this three gradient problem is an affine function (cf. Šverák [13], [14], Zhang [20]) that is to say $\xi+D \varphi$ is identically equal to one of the matrices $\xi_{i}$. Using then the periodicity of $\varphi$ it results that $\xi=\xi_{i} \in E$. We can then conclude that $E$ is quasiconvex.

(iii) Rank one convexity does not imply quasiconvexity. We should again draw the attention to the fact that our result is better for sets than for functions. We prove this assertion in two steps. 
Step 1. There are (cf. Kirchheim-Preiss [7]) $\eta_{1}, \ldots, \eta_{k} \in \partial B(0,1) \subset \mathbb{R}^{2 \times 2}$ such that $\operatorname{rank}\left(\eta_{i}-\eta_{j}\right)=2, \forall i \neq j$ and there is a non affine Lipschitz function $u:(0,1)^{2} \longrightarrow \mathbb{R}^{2}$ with affine boundary data and satisfying $D u(x) \in$ $\left\{\eta_{1}, \ldots, \eta_{k}\right\}$, a.e. in $(0,1)^{2}$.

Step 2. Let $E=\left\{\eta_{1}, \ldots, \eta_{k}\right\}$. Since there are no rank one connections between the matrices $\eta_{i}$, the set $E$ is rank one convex. We will see that $E$ is not quasiconvex. Let $u$ be the function mentioned in Step 1. Since $u$ is Lipschitz and has affine boundary data, we can write $u=u_{\xi}+\varphi$ for some $\varphi \in W_{0}^{1, \infty}\left((0,1)^{2} ; \mathbb{R}^{2}\right)$, denoting by $u_{\xi}$ an affine function such that $D u_{\xi}=\xi$. Besides $D u(x)=\xi+D \varphi(x) \in E$, a.e. in $(0,1)^{2}$, but, as we will see, $\xi \notin E$, which ensures that $E$ is not quasiconvex. We argue by contradiction.

If $\xi \in E$, say $\xi=\eta_{1}$ then, since

$$
\xi=\int_{(0,1)^{2}} D u(x) d x=\sum_{i=1}^{k} \lambda_{i} \eta_{i},
$$

with $\lambda_{i} \in(0,1)$ since $u$ is not affine (in particular $\lambda_{1} \neq 1$ ), we would have

$$
\eta_{1}=\sum_{i=2}^{k} \frac{\lambda_{i}}{1-\lambda_{1}} \eta_{i}
$$

But, since $\eta_{i} \in \partial B(0,1), i=1, \ldots, k$, the above identity is not possible and thus $\xi \neq \eta_{1}$.

(iv) Separate convexity does not imply rank one convexity. Indeed, the set $E=\{\xi, \eta\} \subset \mathbb{R}^{2 \times 2}$, where

$$
\xi=\left(\begin{array}{ll}
2 & 2 \\
0 & 0
\end{array}\right), \quad \eta=\left(\begin{array}{ll}
1 & 1 \\
1 & 1
\end{array}\right)
$$

is separately convex but not rank one convex.

\section{Separation results for polyconvex sets}

We next deal with the problem of separating polyconvex sets generalizing in this way known results in the convex context.

Theorem 13 Let $E$ be a polyconvex set of $\mathbb{R}^{N \times n}$.

(i) If $\eta \notin E$ or $\eta \in \partial E$, then there exists $\beta \in \mathbb{R}^{\tau(N, n)} \backslash\{0\}$ such that

$$
\langle\beta ; T(\eta)-T(\xi)\rangle \leq 0, \forall \xi \in E .
$$

(ii) If $E$ is compact and $\eta \notin E$, then there exists $\beta \in \mathbb{R}^{\tau(N, n)} \backslash\{0\}$ such that

$$
\langle\beta ; T(\eta)\rangle<\inf _{\xi \in E}\{\langle\beta ; T(\xi)\rangle\}
$$


Proof. (i) Since $E$ is polyconvex, if $\eta \notin E$ then $T(\eta) \notin \operatorname{co} T(E)$; in the case $\eta \in \partial E$ then we get $T(\eta) \in \partial \operatorname{co} T(E)$. In both cases, using the separation theorem for convex sets we obtain the existence of $\beta$ satisfying

$$
\langle\beta ; T(\eta)-X)\rangle \leq 0, \forall X \in \operatorname{co} T(E),
$$

and, in particular, for $X \in T(E)$ as desired.

(ii) This stronger result can be obtained using the strong separation theorem for the closed convex set $\operatorname{co} T(E)$.

As a consequence of the previous separation theorem we have the characterization of a polyconvex set given in the following result. This is an extension of the classical version for convex sets which ensures that a closed convex set is the intersection of the closed half-spaces containing the set.

Theorem $14 A$ compact set $E \subset \mathbb{R}^{N \times n}$ is polyconvex if and only if

$$
E=\left\{\xi \in \mathbb{R}^{N \times n}: \varphi(\xi) \geq 0 \text {, for every quasiaffine } \varphi \text { with } \varphi_{\mid E} \geq 0\right\} .
$$

Proof. Let $E$ be a compact polyconvex set and $\xi_{0}$ be such that $\varphi\left(\xi_{0}\right) \geq 0$ for every quasiaffine $\varphi$ satisfying $\varphi_{\mid E} \geq 0$. We will see that $\xi_{0} \in E$. If this was not the case, then, from Theorem 13 (ii),

$$
\left\langle\beta ; T\left(\xi_{0}\right)\right\rangle<c<\inf _{\xi \in E}\{\langle\beta ; T(\xi)\rangle\}
$$

for some $\beta \in \mathbb{R}^{\tau(N, n)} \backslash\{0\}$ and $c \in \mathbb{R}$. Defining $C=c-\inf _{\xi \in E}\{\langle\beta ; T(\xi)\rangle\}$ and the quasiaffine function

$$
\psi(\xi)=\langle\beta ; T(\xi)\rangle+C-\left\langle\beta ; T\left(\xi_{0}\right)\right\rangle
$$

we get a contradiction since $\psi\left(\xi_{0}\right)=C<0$ but, since $\psi_{\mid E} \geq 0$ we should have $\psi\left(\xi_{0}\right) \geq 0$.

The reverse inclusion is evident.

\section{Generalized convex hulls}

Having defined the generalized notions of convexity, we are now in position to introduce the concepts of generalized convex hulls. We follow the same procedure as in the classical convex case.

Definition 15 The polyconvex, quasiconvex, rank one convex and separately convex hulls of a set $E \subset \mathbb{R}^{N \times n}$ are, respectively, the smallest polyconvex, quasiconvex, rank one convex and separately convex sets containing $E$ and are respectively denoted by $\mathrm{Pco} E$, Qco $E$, Rco $E$ and $\operatorname{Sco} E$.

From the discussion made in Section 3, the following inclusions hold:

$$
E \subset \operatorname{Sco} E \subset \operatorname{Rco} E \subset \mathrm{Qco} E \subset \mathrm{P} \operatorname{Po} E \subset \operatorname{co} E \text {. }
$$


As we note below (cf. Remark 25) there are some authors who have adopted other definitions for the rank one convex hull, but this one is more consistent with the convex case. Besides, with the above definitions one has the following result (cf. Dacorogna-Marcellini [5]) whose proof follows in a straightforward manner from Theorem 23 below.

Proposition 16 Let $E$ be a subset of $\mathbb{R}^{N \times n}$ and $\chi_{E}$ be its indicator function. Then

$$
\begin{aligned}
& P \chi_{E}=\chi_{\text {Pco } E} \\
& R \chi_{E}=\chi_{\text {Rco } E} \\
& S \chi_{E}=\chi_{\text {Sco } E}
\end{aligned}
$$

where $P \chi_{E}, R \chi_{E}$ and $S \chi_{E}$ are, respectively, the polyconvex, rank one convex and separately convex envelopes of $\chi_{E}$.

In the following we will give some representations of the hulls defined above. We start giving two characterizations of the polyconvex hull of a set. The second one, which has been proved in Dacorogna-Marcellini [5], is a consequence of Carathéodory theorem and is the equivalent to what is obtained in the convex case.

Theorem 17 Let $E \subset \mathbb{R}^{N \times n}$. Then

(i) $\mathrm{Pco} E=\pi\left(\operatorname{co} T(E) \cap T\left(\mathbb{R}^{N \times n}\right)\right)$,

(ii) $\operatorname{Pco} E=\left\{\xi \in \mathbb{R}^{N \times n}: T(\xi)=\sum_{i=1}^{\tau+1} \lambda_{i} T\left(\xi_{i}\right), \xi_{i} \in E,\left(\lambda_{1}, \ldots, \lambda_{\tau+1}\right) \in \Lambda_{\tau+1}\right\}$.

In particular, if $E$ is compact, then $\mathrm{Pco} E$ is also compact and if $E$ is open, then Pco $E$ is also open.

Proof. (i) We prove the first representation of Pco $E$. It is clear that Pco $E \subset$ $\pi\left(\operatorname{co} T(E) \cap T\left(\mathbb{R}^{N \times n}\right)\right)$. For the other inclusion we start noting that, since Pco $E$ is polyconvex, by definition,

$$
\operatorname{Pco} E=\pi\left(K \cap T\left(\mathbb{R}^{N \times n}\right)\right)
$$

for some convex set $K \subset \mathbb{R}^{\tau(N, n)}$. Since $E \subset \operatorname{Pco} E, K$ must contain $T(E)$ and, consequently, must contain $\operatorname{co} T(E)$, from that the desired inclusion follows.

(ii) For this second representation of Pco $E$, denoting by $Y$ the set on the right hand side, it immediately follows, from the definition of polyconvex set, that $Y \subset \operatorname{Pco} E$. Moreover, one easily verifies that $Y$ is a polyconvex set containing $E$ which implies that Pco $E \subset Y$.

For the assertion concerning compact sets, it is trivial that Pco $E$ is bounded if $E$ is compact. Let then $\xi_{\nu} \in \operatorname{Pco} E$ with $\xi_{\nu} \rightarrow \xi$. By the first representation of Pco $E, T\left(\xi_{\nu}\right) \in \operatorname{co} T(E)$, which is a compact set since $T(E)$ is compact. Then $T(\xi)=\lim T\left(\xi_{\nu}\right) \in \operatorname{co} T(E)$ and thus $\xi \in$ Pco $E$ as wished. 
Finally, it can be seen, using an inductive argument, that, if

$$
T(\xi)=\sum_{i=1}^{\tau+1} \lambda_{i} T\left(\xi_{i}\right),
$$

for some $\xi, \xi_{i} \in \mathbb{R}^{N \times n}$ and $\left(\lambda_{1}, \ldots, \lambda_{\tau+1}\right) \in \Lambda_{\tau+1}$, then

$$
T(\xi+\eta)=\sum_{i=1}^{\tau+1} \lambda_{i} T\left(\xi_{i}+\eta\right), \forall \eta \in \mathbb{R}^{N \times n} .
$$

From this and (ii), it easily follows that Pco $E$ is open if $E$ is open.

We now give a different representation of the polyconvex hull, using the separation results of the previous section.

Theorem 18 Let $E \subset \mathbb{R}^{N \times n}$ be such that Pco $E$ is compact. Then

$$
\text { Pco } E=\left\{\xi \in \mathbb{R}^{N \times n}: \varphi(\xi) \geq 0 \text {, for every quasiaffine } \varphi \text { with } \varphi_{\mid E} \geq 0\right\} \text {. }
$$

Proof. The set in the right hand side is polyconvex and contains $E$, then it contains Pco $E$. On the other hand, since Pco $E$ is polyconvex and compact then, by Theorem 13 we have

$$
\text { Pco } E=\left\{\xi \in \mathbb{R}^{N \times n}: \varphi(\xi) \geq 0 \text {, for every quasiaffine } \varphi \text { with } \varphi_{\mid \mathrm{Pco} E} \geq 0\right\} .
$$

Since any quasiaffine function $\varphi$ with $\varphi_{\mid \operatorname{Pco} E} \geq 0$ verifies also $\varphi_{\mid E} \geq 0$, one gets

$$
\left\{\xi \in \mathbb{R}^{N \times n}: \varphi(\xi) \geq 0, \text { for every quasiaffine } \varphi \text { with } \varphi_{\mid E} \geq 0\right\} \subset \operatorname{Pco} E,
$$

which finishes the proof.

We next give a representation for the quasiconvex hull, similar to (ii) of Theorem 17. This representation is however weaker than the one obtained in the polyconvex case since we cannot obtain the representation formula in a prescribed finite number of steps.

Theorem 19 Let $E \subset \mathbb{R}^{N \times n}$. Let $\mathrm{Q}_{0} \operatorname{co} E=E$ and define by induction the sets

$$
\mathrm{Q}_{i+1} \operatorname{co} E=\left\{\xi \in \mathbb{R}^{N \times n}: \begin{array}{c}
\exists R \in O(n), \varphi \in \mathcal{W}_{R} \text { such that } \\
\xi+D \varphi(x) \in \mathrm{Q}_{i} \operatorname{co} E, \text { a.e. } x \in R \Omega
\end{array}\right\}, i \geq 0 .
$$

Then Qco $E=\cup_{i \in \mathbb{N}} Q_{i} \operatorname{co} E$.

In particular, if $E$ is open, then Qco $E$ is also open.

Proof. By definition of quasiconvex set and by induction, we have $\mathrm{Q}_{i} \operatorname{co} E \subset$ Qco $E$, for every $i$ and thus $\cup_{i \in \mathbb{N}} Q_{i} \operatorname{co} E \subset$ Qco $E$. The reverse inclusion follows from the fact that $\cup_{i \in \mathbb{N}} Q_{i} \operatorname{co} E$ is, as we will see, a quasiconvex set. 
Let $R \in O(n), \varphi \in \mathcal{W}_{R}$ and $\xi+D \varphi(x) \in \cup_{i \in \mathbb{N}} Q_{i} \operatorname{co} E$, a.e. $x \in R \Omega$. One has

$$
\begin{gathered}
D \varphi(x) \in\left\{\eta_{1}, \ldots, \eta_{k}\right\} \text { a.e. } x \in R \Omega, \text { with } \\
\operatorname{meas}\left\{x \in R \Omega: D \varphi(x)=\eta_{i}\right\}>0, i=1, \ldots, k .
\end{gathered}
$$

Moreover, $\xi+\eta_{i} \in \mathrm{Q}_{\alpha(i)} \operatorname{co} E$ for some $\alpha(i) \in \mathbb{N}$. Let $s=\max \{\alpha(1), \ldots, \alpha(k)\}$. Since $\mathrm{Q}_{i} \operatorname{co} E \subset \mathrm{Q}_{i+1} \operatorname{co} E$, we have, for all $i=1, \ldots, k, \xi+\eta_{i} \in \mathrm{Q}_{s} \operatorname{co} E$. Thus $\xi+D \varphi(x) \in \mathrm{Q}_{s} \operatorname{co} E$ and, by definition, we get $\xi \in \mathrm{Q}_{s+1} \operatorname{co} E \subset \cup_{i \in \mathbb{N}} \mathrm{Q}_{i} \operatorname{co} E$; the quasiconvexity of this last set follows.

Under the hypothesis of $E$ being an open set, one easily gets, using induction arguments, that each $\mathrm{Q}_{i} \mathrm{co} E$ is open. By the preceding representation of Qco $E$ it follows that this set is also open.

The analogous representation for the rank one convex hull of a set is given in the result below (for the proof, see Dacorogna-Marcellini [5, page 136]).

Theorem 20 Let $E \subset \mathbb{R}^{N \times n}$. Let $\mathrm{R}_{0} \operatorname{co} E=E$ and define by induction the sets

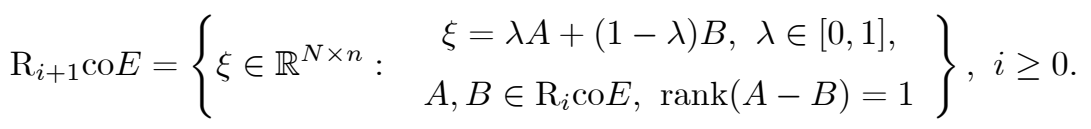

Then $\operatorname{Rco} E=\cup_{i \in \mathbb{N}} \mathrm{R}_{i} \operatorname{co} E$.

In particular, if $E$ is open, then $\operatorname{Rco} E$ is also open.

Remark 21 (i) Similar construction and results can be obtained for Sco E.

(ii) The last assertion of the theorem follows, as in the quasiconvex case, from the fact that each $\mathrm{R}_{i} \operatorname{co} E$ is open if $E$ itself is open.

(iii) In general it is not true that rank one convex hulls or separately convex hulls of compact sets are compact (see Aumann-Hart [1] and Kolár [9]).

We will now consider representations of the convex hulls through functions as we can get in the convex case.

Notation 22 Given a set $E \subset \mathbb{R}^{N \times n}$, we consider the following sets of functions

$$
\begin{aligned}
\overline{\mathcal{F}}_{E} & =\left\{f: \mathbb{R}^{N \times n} \rightarrow \mathbb{R} \cup\{+\infty\}:\left.f\right|_{E} \leq 0\right\} \\
\mathcal{F}_{E} & =\left\{f: \mathbb{R}^{N \times n} \rightarrow \mathbb{R}:\left.f\right|_{E} \leq 0\right\} .
\end{aligned}
$$

With the above notation, one has, for $E \subset \mathbb{R}^{N \times n}$,

$$
\begin{aligned}
& \operatorname{co} E=\left\{\xi \in \mathbb{R}^{N \times n}: f(\xi) \leq 0, \text { for every convex } f \in \overline{\mathcal{F}}_{E}\right\} \\
& \overline{\operatorname{co} E}=\left\{\xi \in \mathbb{R}^{N \times n}: f(\xi) \leq 0, \text { for every convex } f \in \mathcal{F}_{E}\right\}
\end{aligned}
$$

where $\overline{\operatorname{co} E}$ denotes the closure of the convex hull of $E$.

Analogous representations to (??) can be obtained in the polyconvex, rank one convex and separately convex cases. However, (4) can only be generalized to the polyconvex case if the sets are compact (see Theorem 26). When dealing with the other notions of convexity, (4) is not true, even if compact sets are considered. 
Theorem 23 Let $E \subset \mathbb{R}^{N \times n}$, then

$$
\begin{aligned}
\text { Pco } E & =\left\{\xi \in \mathbb{R}^{N \times n}: f(\xi) \leq 0, \text { for every polyconvex } f \in \overline{\mathcal{F}}_{E}\right\} \\
\text { Rco } E & =\left\{\xi \in \mathbb{R}^{N \times n}: f(\xi) \leq 0, \text { for every rank one convex } f \in \overline{\mathcal{F}}_{E}\right\} \\
\text { Sco } E & =\left\{\xi \in \mathbb{R}^{N \times n}: f(\xi) \leq 0, \text { for every separately convex } f \in \overline{\mathcal{F}}_{E}\right\} .
\end{aligned}
$$

Proof. We prove the first identity, the others being analogous. Let us call $X$ the set in the right hand side. Evidently $X$ is a polyconvex set containing $E$ and thus Pco $E \subset X$. Consider now $\xi \in X$. Since $\chi_{\mathrm{Pco} E}$ is a polyconvex function of $\overline{\mathcal{F}}_{E}$, one has $\chi_{\mathrm{Pco} E}(\xi) \leq 0$ and consequently $\xi \in \operatorname{Pco} E$ obtaining the other inclusion.

We next introduce some new sets which will allow a better understanding of the closure of the different hulls.

Definition 24 For a set $E$ of $\mathbb{R}^{N \times n}$, let

$$
\begin{aligned}
\operatorname{co}_{f} E & =\left\{\xi \in \mathbb{R}^{N \times n}: f(\xi) \leq 0, \text { for every convex } f \in \mathcal{F}_{E}\right\} \\
\operatorname{Pco}_{f} E & =\left\{\xi \in \mathbb{R}^{N \times n}: f(\xi) \leq 0, \text { for every polyconvex } f \in \mathcal{F}_{E}\right\} \\
\operatorname{Qco}_{f} E & =\left\{\xi \in \mathbb{R}^{N \times n}: f(\xi) \leq 0, \text { for every quasiconvex } f \in \mathcal{F}_{E}\right\} \\
\operatorname{Rco}_{f} E & =\left\{\xi \in \mathbb{R}^{N \times n}: f(\xi) \leq 0, \text { for every rank one convex } f \in \mathcal{F}_{E}\right\} \\
\operatorname{Sco}_{f} E & =\left\{\xi \in \mathbb{R}^{N \times n}: f(\xi) \leq 0, \text { for every separately convex } f \in \mathcal{F}_{E}\right\} .
\end{aligned}
$$

Remark 25 (i) As well known,

$$
\operatorname{co}_{f} E=\overline{\operatorname{co} E} .
$$

(ii) The above sets are all closed because any separately convex function taking only finite values is continuous. Besides, they are, respectively, (according to our definitions) convex, polyconvex, quasiconvex, rank one convex and separately convex.

(iii) Some authors (see, for example, Müller-Šverák [12], Šverák [16], Zhang [19]), when dealing with quasiconvexity and rank one convexity, have adopted the above definitions for the hull of a set (in the generalized senses). They call laminate convex hull what we have called Rco $E$.

(iv) As in Theorem 17, it can easily be shown that

$$
\mathrm{Pco}_{f} E=\pi\left(\operatorname{co}_{f} T(E) \cap T\left(\mathbb{R}^{N \times n}\right)\right) .
$$

We next see the relations between the closures of the convex hulls and the sets introduced in the above definition.

Theorem 26 Given any set $E \subset \mathbb{R}^{N \times n}$ and denoting by $\overline{\mathrm{P}} \mathrm{co} E$, $\overline{\mathrm{Q} c o} E$, $\overline{\mathrm{R}} \mathrm{E} E$ and $\overline{\mathrm{Sco} E}$ the closure of, respectively, the polyconvex, quasiconvex, rank one 
convex and separately convex hulls of $E$, we have

$$
\begin{aligned}
& \overline{\mathrm{Pco} E} \subset \overline{\mathrm{Pco}_{f} E} \\
& \overline{\mathrm{Qco} E} \subset \mathrm{Qco}_{f} E \\
& \overline{\operatorname{Rco} E} \subset \mathrm{Rco}_{f} E \\
& \overline{\operatorname{Sco} E} \subset \mathrm{Sco}_{f} E .
\end{aligned}
$$

In general, the four inclusions are strict. However if $E$ is compact, then

$$
\operatorname{Pco} E=\overline{\mathrm{Pco} E}=\mathrm{Pco}_{f} E .
$$

Remark 27 We call the attention to the fact that, contrary to what was stated in Dacorogna-Marcellini [5, page 132], in general, $\overline{\mathrm{Pco} E} \neq \mathrm{Pco}_{f} E$, unless $E$ is compact. We should also draw the attention (cf. Proposition 28) that in general the sets $\overline{\mathrm{P} c o} E, \overline{\mathrm{Q} c o} E, \overline{\mathrm{Rco} E}, \overline{\mathrm{Sco} E}$ are not even separately convex.

Proof. Since $\mathrm{Pco}_{f} E$ is a closed polyconvex set containing $E$ then $\overline{\mathrm{Pco} E} \subset$ $\mathrm{Pco}_{f} E$. In the same way we get the inclusions for the quasiconvex, rank one convex and separately convex cases.

We now deal with the fact that the inclusions are strict. The first one follows (cf. Proposition 28 below) from the fact that there are polyconvex sets whose closure is not polyconvex though $\mathrm{Pco}_{f} E$ is always a polyconvex set. If we assume $E$ to be compact then we have, as we will see,

$$
\mathrm{P} c o E=\overline{\mathrm{Pco} E}=\mathrm{Pco}_{f} E \text {. }
$$

By Theorem 17, in this case, Pco $E$ is compact and then Pco $E=\overline{\mathrm{Pco} E}$. We will prove that $\mathrm{Pco}_{f} E \subset \mathrm{Pco} E$. We start noting that, since $E$ is compact, $T(E)$ is compact and thus co $T(E)$ is also compact. Considering $\xi \in \mathrm{Pco}_{f} E$ then, since the function $\eta \mapsto \operatorname{dist}(T(\eta), \operatorname{co} T(E))$ is a polyconvex function, $\operatorname{dist}(T(\xi), \operatorname{co} T(E))=0$. Since $\operatorname{co} T(E)$ is closed, we can deduce that $T(\xi) \in$ $\operatorname{co} T(E)$ and thus, $\xi \in \mathrm{P} \operatorname{co} E$.

Next we use an example due to Casadio [2] (or equivalent examples by Aumann-Hart [1] and Tartar [17]) which will give at once $\overline{\mathrm{Qco} E} \underset{\neq}{\subsetneq} \mathrm{Qco}_{f} E$, $\overline{\operatorname{Rco} E} \underset{\neq}{\subset} \operatorname{Rco}_{f} E$ and $\overline{\operatorname{Sco} E} \underset{f}{\subset} \operatorname{Sco}_{f} E$. The second non inclusion was already observed in Dacorogna-Marcellini [5, page 133]. Consider the following four diagonal matrices of $\mathbb{R}^{2 \times 2}$

$$
\xi_{1}=\operatorname{diag}(-1,0), \xi_{2}=\operatorname{diag}(1,-1), \xi_{3}=\operatorname{diag}(2,1), \xi_{4}=\operatorname{diag}(0,2) .
$$

Since $\operatorname{rank}\left(\xi_{i}-\xi_{j}\right)=2$ for $i \neq j$, the set $E=\left\{\xi_{1}, \xi_{2}, \xi_{3}, \xi_{4}\right\}$ is rank one convex. It is also quasiconvex, the argument is the same as in the proof of Theorem 11, assertion (ii) of Part 2, here using the non existence of non-affine Lipschitz functions whose gradient takes four possible values with no rank one connections (cf. Chlebík-Kirchheim [3]). However, any separately convex function $f \in \mathcal{F}_{E}$ 
and consequently any rank one convex or quasiconvex function in $\mathcal{F}_{E}$, has $f(0) \leq$ 0 (see [5]). Thus $0 \in \operatorname{Sco}_{f} E$, but $0 \notin \overline{\mathrm{Qco} E}$.

We can write

$$
\overline{\mathrm{Sco} E} \subset \overline{\mathrm{Rco} E} \subset \overline{\mathrm{Qco} E} \subset \overline{\mathrm{P} c o} E \subset \overline{\operatorname{co} E}=\operatorname{co}_{f} E
$$

and also

$$
\mathrm{Sco}_{f} E \subset \mathrm{Rco}_{f} E \subset \mathrm{Qco}_{f} E \subset \mathrm{Pco}_{f} E \subset \overline{\operatorname{co} E}=\operatorname{co}_{f} E .
$$

Moreover, the same example and arguments used in the proof of Theorem 26 (see also Proposition 28) shows that, in general,

$$
\operatorname{Sco}_{f} E \nsubseteq \overline{\operatorname{Rco} E}, \quad \operatorname{Rco}_{f} E \nsubseteq \overline{\mathrm{Qco}} \quad \text { and } \quad \mathrm{Qco}_{f} E \nsubseteq \overline{\mathrm{Pco} E} \text {. }
$$

However, if $E$ is compact one has $\mathrm{Qco}_{f} E \subset \overline{\mathrm{Pco} E}$.

We draw the attention to the fact that several characterizations of the sets in Definition 24 have been used in the literature according to the specific needs of each situation. These sets can be written in terms of measures (cf. Kirchheim [8], Müller [11]) or using the distance function (cf. Zhang [18]): if $E \subset \mathbb{R}^{N \times n}$ is compact, then

$$
\mathrm{Qco}_{f} E=\left\{\xi \in \mathbb{R}^{N \times n}: Q \operatorname{dist}(\xi, E)=0\right\},
$$

where $Q \operatorname{dist}(\cdot, E)$ is the quasiconvex envelope of the function $\operatorname{dist}(\cdot, E)$.

We next prove, as already mentioned in Remark 12, that the interior of generalized convex sets keeps the convexity (in the generalized sense), but that, contrary to the classical convex case, this is not true for the closure.

Proposition 28 (i) Let $E \subset \mathbb{R}^{N \times n}$ be, respectively, a polyconvex, quasiconvex, rank one convex or separately convex set. Then int $E$ is also, respectively, polyconvex, quasiconvex, rank one convex or separately convex.

(ii) There is $E \subset \mathbb{R}^{2 \times 2}$ a polyconvex and bounded set such that $\bar{E}$ is not separately convex.

Proof. (i) We present the proof in the context of polyconvexity. For the other convexities the proof is analogous. It is sufficient to prove that $\operatorname{Pco}(\operatorname{int} E)=$ $\operatorname{int} E$. The non trivial inclusion is $\operatorname{Pco}(\operatorname{int} E) \subset \operatorname{int} E$. Since $E$ is polyconvex, evidently

$$
\operatorname{Pco}(\text { int } E) \subset \operatorname{Pco} E=E .
$$

On the other hand, int $E$ is open and thus (cf. Theorem 17) Pco(int $E$ ) is also open. From (6), it follows then the desired inclusion.

(ii) We define

$$
E=\left\{ \pm\left(\begin{array}{ll}
1 & 0 \\
0 & x
\end{array}\right): 0<x<1\right\}
$$


It is a bounded set and $\bar{E}$ is not separately convex. In fact, let $\xi_{1}=\operatorname{diag}(1,0)$ and $\xi_{2}=\operatorname{diag}(-1,0)$, one has $\xi_{1}, \xi_{2} \in \bar{E}$, but $\lambda \xi_{1}+(1-\lambda) \xi_{2} \notin \bar{E}$ for any $0<\lambda<1$.

We now show that $E$ is polyconvex. Let $\xi_{1}, \ldots, \xi_{6} \in E$ and suppose

$$
T(\xi)=\sum_{i=1}^{6} \lambda_{i} T\left(\xi_{i}\right) \text {, for some }\left(\lambda_{1}, \ldots, \lambda_{6}\right) \in \Lambda_{6} .
$$

We have to see that $\xi \in E$. We can write $\{1, \ldots, 6\}=I_{+} \cup I_{-}$for some $I_{+}$and $I_{-}$such that

$$
\xi_{i}=\left(\begin{array}{cc}
1 & 0 \\
0 & x_{i}
\end{array}\right) \text { if } i \in I_{+} \text {and } \xi_{i}=\left(\begin{array}{cc}
-1 & 0 \\
0 & -x_{i}
\end{array}\right) \text { if } i \in I_{-},
$$

where $0<x_{i}<1, i=1, \ldots, 6$. In any case $\operatorname{det} \xi_{i}=x_{i}$.

If $I_{+}=\emptyset$ or $I_{-}=\emptyset$ then it is clear that $\xi \in E$. We will see that the other case: $I_{+} \neq \emptyset$ and $I_{-} \neq \emptyset$, is not an admissible one. In fact, from (7), we can write

$$
\xi=\left(\begin{array}{cc}
\sum_{i \in I_{+}} \lambda_{i}-\sum_{i \in I_{-}} \lambda_{i} & 0 \\
0 & \sum_{i \in I_{+}} \lambda_{i} x_{i}-\sum_{i \in I_{-}} \lambda_{i} x_{i}
\end{array}\right)=\left(\begin{array}{cc}
\alpha & 0 \\
0 & \beta
\end{array}\right)
$$

and $\operatorname{det} \xi=\alpha \beta=\sum_{i=1}^{6} \lambda_{i} x_{i}$.

Then $|\alpha|<\sum_{i=1}^{6} \lambda_{i}=1,|\beta|<\sum_{i=1}^{6} \lambda_{i} x_{i}$ and thus $|\alpha \beta|<\sum_{i=1}^{6} \lambda_{i} x_{i}$, which is a contradiction.

\section{Extreme points}

An important tool in convex analysis is the notion of extreme point. In a straightforward manner we can define it for generalized convex sets as follows (cf. Dacorogna-Marcellini [5, page 138]).

Definition 29 (i) If $E \subset \mathbb{R}^{m}$ is convex, $\xi \in E$ is said to be an extreme point of $E$ in the convex sense if

$$
\left.\begin{array}{c}
\xi=\lambda \xi_{1}+(1-\lambda) \xi_{2} \\
\lambda \in(0,1), \xi_{1}, \xi_{2} \in E
\end{array}\right\} \Rightarrow \xi_{1}=\xi_{2}=\xi
$$

For an arbitrary set $E \subset \mathbb{R}^{m}$, the set of extreme points of $\operatorname{co} E$ will be denoted $E_{\text {ext }}^{c}$. 
(ii) If $E \subset \mathbb{R}^{N \times n}$ is polyconvex, $\xi \in E$ is said to be an extreme point of $E$ in the polyconvex sense if

$$
\left.\begin{array}{c}
T(\xi)=\sum_{i=1}^{\tau+1} \lambda_{i} T\left(\xi_{i}\right), \\
\left.\lambda_{\tau+1}\right) \in \Lambda_{\tau+1}, \lambda_{i}>0, \xi_{i} \in E
\end{array}\right\} \Rightarrow \xi_{i}=\xi, i=1, \ldots, \tau+1
$$

For an arbitrary set $E \subset \mathbb{R}^{N \times n}$, the set of extreme points of Pco $E$ will be denoted $E_{\text {ext }}^{p}$.

(iii) If $E \subset \mathbb{R}^{N \times n}$ is quasiconvex, $\xi \in E$ is said to be an extreme point of $E$ in the quasiconvex sense if

$$
\left.\begin{array}{c}
\xi+D \varphi(x) \in E, \text { a.e. } x \in R \Omega, \\
\Omega=(0,1)^{n}, R \in O(n), \varphi \in \mathcal{W}_{R}
\end{array}\right\} \Rightarrow D \varphi \equiv 0 .
$$

For an arbitrary set $E \subset \mathbb{R}^{N \times n}$, the set of extreme points of Qco $E$ will be denoted $E_{\text {ext }}^{q}$.

(iv) If $E \subset \mathbb{R}^{N \times n}$ is rank one convex, $\xi \in E$ is said to be an extreme point of $E$ in the rank one convex sense if

$$
\left.\begin{array}{c}
\xi=\lambda \xi_{1}+(1-\lambda) \xi_{2} \\
\lambda \in(0,1), \xi_{1}, \xi_{2} \in E, \operatorname{rank}\left(\xi_{1}-\xi_{2}\right) \leq 1
\end{array}\right\} \Rightarrow \xi_{1}=\xi_{2}=\xi
$$

For an arbitrary set $E \subset \mathbb{R}^{N \times n}$, the set of extreme points of Rco $E$ will be denoted $E_{\text {ext }}^{r}$.

(v) If $E \subset \mathbb{R}^{m}$ is separately convex, $\xi \in E$ is said to be an extreme point of $E$ in the separately convex sense if

$$
\left.\begin{array}{c}
\xi=\lambda \xi_{1}+(1-\lambda) \xi_{2} \\
\lambda \in(0,1), \xi_{1}, \xi_{2} \in E, \xi_{1}-\xi_{2}=s e_{i}, \\
\text { with } s \in \mathbb{R} \text { and } e_{i} \text { a vector of the canonical basis of } \mathbb{R}^{m}
\end{array}\right\} \Rightarrow \xi_{1}=\xi_{2}=\xi
$$

For an arbitrary set $E \subset \mathbb{R}^{m}$, the set of extreme points of Sco $E$ will be denoted $E_{\text {ext }}^{s}$.

We next see the relations between the sets of extreme points for the different notions of convexity.

Proposition 30 Let $E \subset \mathbb{R}^{N \times n}$. Then

$$
E_{\text {ext }}^{c} \subset E_{\text {ext }}^{p} \subset E_{\text {ext }}^{q} \subset E_{\text {ext }}^{r} \subset E_{\text {ext }}^{s} .
$$

Proof. The non trivial inclusions are those related to $E_{\text {ext }}^{q}$, the set of extreme points of Qco $E$, but it can be obtained with the same arguments used in the proof of Theorem 11, Part 1, and we opt not to repeat them. 
Minkowski theorem (often better known as Krein-Milman theorem which is its infinite dimensional version) assures that the convex hull of a compact set coincides with the convex hull of its extreme points. We next deal with the generalization of this result to the other convexities. We start with the polyconvex case (see also Dacorogna-Tanteri [6]).

Theorem 31 Let $E \subset \mathbb{R}^{N \times n}$ be a compact set. Then

$$
\operatorname{Pco} E=\operatorname{Pco} E_{e x t}^{p} \text {. }
$$

Proof. One inclusion is trivial: Pco $E_{\text {ext }}^{p} \subset \mathrm{Pco} E$, since $E_{\text {ext }}^{p} \subset \mathrm{Pco} E$. We will next show the reverse inclusion. We start remarking that

$$
\begin{aligned}
& \text { Pco } E=\pi\left(\operatorname{co} T(E) \cap T\left(\mathbb{R}^{N \times n}\right)\right) \\
& \text { Pco } E_{\text {ext }}^{p}=\pi\left(\operatorname{co} T\left(E_{\text {ext }}^{p}\right) \cap T\left(\mathbb{R}^{N \times n}\right)\right) .
\end{aligned}
$$

Let $\xi \in \mathrm{P} c o E$. We will see that $\xi \in \mathrm{P} c o E_{\text {ext }}^{p}$. By the above characterization of Pco $E$ we have $T(\xi) \in \operatorname{co} T(E)$. Moreover, by Minkowski theorem, and using the fact that $T(E)$ is compact, we have

$$
\operatorname{co} T(E)=\operatorname{co}\left(T(E)_{e x t}^{c}\right),
$$

where $T(E)_{e x t}^{c}$ is the set of extreme points of co $T(E)$ (in the convex sense).

We will next prove that

$$
T(E)_{e x t}^{c} \subset T\left(E_{e x t}^{p}\right),
$$

which will finish the proof.

Let then $X \in T(E)_{e x t}^{c}$. In particular, $X \in T(E)$ and we can write $X=T(\eta)$ with $\eta \in E$. It suffices then to see that $\eta \in E_{\text {ext }}^{p}$. Suppose that

$$
T(\eta)=\sum_{i=1}^{\tau+1} \lambda_{i} T\left(\eta_{i}\right)
$$

for some $\left(\lambda_{1}, \ldots, \lambda_{\tau+1}\right) \in \Lambda_{\tau+1}, \quad \lambda_{i}>0, \eta_{i} \in \operatorname{Pco} E$. Noting that, since $\eta_{i} \in$ Pco $E$ then $T\left(\eta_{i}\right) \in \operatorname{co} T(E)$, it immediately follows, from the fact that $T(\eta)$ is an extreme point of $\operatorname{co} T(E)$, that $\eta_{i}=\eta$ for every $i$, that is to say $\eta$ is an extreme point of Pco $E$. The proof is finished.

As remarked in Kirchheim [8], the result above is not true for quasiconvex, rank one convex or separately convex hulls (see Example 33 below). Even though, for these cases, a weaker result can be proved (cf. Theorem 32). We reproduce the proof of Matoušek-Plecháč [10], which is also seen to apply to the quasiconvex case. See also Zhang [18] for the quasiconvex case.

Theorem 32 Let $E \subset \mathbb{R}^{N \times n}$ be a bounded set and $E_{\text {ext }}^{q f}$, $E_{\text {ext }}^{r f}$, $E_{\text {ext }}^{s f}$ denote, respectively, the set of extreme points of $\mathrm{Qco}_{f} E$ (in the quasiconvex sense), the set of extreme points of $\mathrm{Rco}_{f} E$ (in the rank one convex sense) and the set of extreme points of $\mathrm{Sco}_{f} E$ (in the separately convex sense). Then

$\mathrm{Qco}_{f} E=\mathrm{Qco}_{f} E_{e x t}^{q f} \quad \operatorname{Rco}_{f} E=\operatorname{Rco}_{f} E_{e x t}^{r f} \quad$ and $\quad \operatorname{Sco}_{f} E=\operatorname{Sco}_{f} E_{e x t}^{s f}$. 
Proof. We divide the proof in two steps. The first is common to the three convexities and we present it in the context of quasiconvexity. In the second step we consider separately the quasiconvex and the rank one convex cases (this last being analogous to the separately convex case). In all what follows we will denote by $\bar{E}_{e x t}^{q f}$ the closure of $E_{e x t}^{q f}$

Step 1. We remark that, for any set $K \subset \mathbb{R}^{N \times n}$, since $\mathrm{Qco}_{f}$ is automatically closed, $\mathrm{Qco}_{f} K=\mathrm{Qco}_{f} \bar{K}$. Thus, it is enough to prove that $\mathrm{Qco}_{f} E=$ $\mathrm{Qco}_{f} \bar{E}_{e x t}^{q f}$. The inclusion $\mathrm{Qco}_{f} \bar{E}_{e x t}^{q f} \subset \mathrm{Qco}_{f} E$ is trivial. It remains to verify the reverse inclusion. We use a contradiction argument.

Suppose there is some $\eta \in \mathrm{Qco}_{f} E \backslash \mathrm{Qco}_{f} \bar{E}_{e x t}^{q f}$, then, by definition, there exists a quasiconvex function $f: \mathbb{R}^{N \times n} \longrightarrow \mathbb{R}$ with $f \in \mathcal{F}_{\bar{E}_{e x t}^{q f}}$, such that $f(\eta)>0$.

Now let

$$
M=\max _{\mathrm{Qco}_{f} E} f \quad \text { and } \quad \mathcal{A}=\left\{\xi \in \mathrm{Qco}_{f} E: f(\xi)=M\right\} .
$$

This set is nonempty and compact (since $\mathrm{Qco}_{f} E$ is compact and $f$ is a continuous function). Thus, considering $\mathbb{R}^{N \times n}$ with the lexicographic order (the elements of $\mathbb{R}^{N \times n}$ being seen as vectors) one can consider the maximum element of $\mathcal{A}$, say $\xi_{0}$. We have $\xi_{0} \notin E_{e x t}^{q f}$, which follows from

$$
0<f(\eta) \leq \max _{\mathrm{Qco}_{f} E} f=M=f\left(\xi_{0}\right) .
$$

As we will see in Step 2 this will lead to the existence of an element in $\mathcal{A}$ greater than $\xi_{0}$ for the lexicographic order, which is absurd.

Step 2. Quasiconvex case. Since $\xi_{0} \in \mathrm{Qco}_{f} E \backslash E_{\text {ext }}^{q f}$, there are $R \in O(n)$ and $\varphi \in \mathcal{W}_{R}$ such that

$$
\xi_{0}+D \varphi(x) \in \mathrm{Qco}_{f} E, \text { a.e. } x \in R \Omega \text {, with } D \varphi \not \equiv 0 .
$$

We can write

$$
D \varphi(x) \in\left\{\xi_{1}, \ldots, \xi_{k}\right\} \text { and } \lambda_{i}=\operatorname{meas}\left\{x \in R \Omega: D \varphi(x)=\xi_{i}\right\}>0 .
$$

Since $\xi_{0}+\xi_{i} \in \mathrm{Qco}_{f} E$, we have $f\left(\xi_{0}+\xi_{i}\right) \leq M$. Consequently, by the quasiconvexity of $f$ we get

$$
M=f\left(\xi_{0}\right) \leq \int_{R \Omega} f\left(\xi_{0}+D \varphi(x)\right) d x=\sum_{i=1}^{k} \lambda_{i} f\left(\xi_{0}+\xi_{i}\right) \leq M
$$

implying $f\left(\xi_{0}+\xi_{i}\right)=M, i=1, \ldots, k$ that is $\xi_{0}+\xi_{i} \in \mathcal{A}$. Finally, from the fact that $D \varphi \not \equiv 0$ and $0=\int_{R \Omega} D \varphi(x) d x=\sum_{i=1}^{k} \lambda_{i} \xi_{i}$ we conclude that among the elements $\xi_{0}+\xi_{i}$ there must be at least one which is greater than $\xi_{0}$ (in the lexicographic order) which contradicts the fact that $\xi_{0}$ is the maximum element of $\mathcal{A}$. 
Rank one convex case. We recall that in this case the function $f$ is a rank one convex function. Since $\xi_{0} \in \operatorname{Rco}_{f} E \backslash E_{e x t}^{r f}$, there are $\eta_{1}, \eta_{2} \in \operatorname{Rco}_{f} E$, with $\operatorname{rank}\left(\eta_{1}-\eta_{2}\right) \leq 1$ such that $\xi_{0}=\lambda \eta_{1}+(1-\lambda) \eta_{2}$ and $\xi_{0} \neq \eta_{1}, \xi_{0} \neq \eta_{2}$. As in the quasiconvex case we get $f\left(\eta_{1}\right)=f\left(\eta_{2}\right)=M$ and from $\xi_{0}=\lambda \eta_{1}+(1-\lambda) \eta_{2}$ it follows that $\eta_{1}$ or $\eta_{2}$ must be greater than $\xi_{0}$, which is a contradiction.

As observed by Kirchheim [8], the example of Casadio [2] (or those of Aumann-Hart [1] and Tartar [17]) considered in the proof of Theorem 26 shows that, in general,

$$
\mathrm{Qco} E_{\text {ext }}^{q} \neq \mathrm{Qco} E, \quad \mathrm{Rco} E_{\text {ext }}^{r} \neq \mathrm{R} \operatorname{Rco} E \quad \text { and } \quad \operatorname{Sco} E_{\text {ext }}^{s} \neq \operatorname{Sco} E .
$$

Example 33 We consider a set of diagonal matrices which we identify with elements of $\mathbb{R}^{2}$. In particular, rank one convexity and separate convexity coincide.

Let

$$
E=E_{1} \cup E_{2} \cup E_{3} \cup E_{4} \cup E_{5},
$$

where

$$
\begin{aligned}
& E_{1}=\left\{(x, y) \in \mathbb{R}^{2}: 0 \leq x \leq 1,0 \leq y \leq 1\right\}, \\
& E_{2}=\left\{(x, 1) \in \mathbb{R}^{2}: 1 \leq x \leq 2\right\}, \quad E_{3}=\left\{(0, y) \in \mathbb{R}^{2}: 1 \leq y \leq 2\right\}, \\
& E_{4}=\left\{(x, 0) \in \mathbb{R}^{2}: \quad-1 \leq x \leq 0\right\}, \quad E_{5}=\left\{(1, y) \in \mathbb{R}^{2}:-1 \leq y \leq 0\right\} .
\end{aligned}
$$

Note that $E$ is a compact rank one convex set and

$$
E_{\text {ext }}^{q} \subset E_{\text {ext }}^{r}=\left\{\xi_{1}, \xi_{2}, \xi_{3}, \xi_{4}\right\},
$$

where

$$
\xi_{1}=(-1,0), \quad \xi_{2}=(1,-1), \xi_{3}=(2,1), \xi_{4}=(0,2)
$$

Thus, since there are no rank one connections between the elements $\xi_{i}$, Qco $E_{\text {ext }}^{q}=E_{\text {ext }}^{q}$ and Rco $E_{\text {ext }}^{r}=E_{\text {ext }}^{r}$. However, $E_{\text {ext }}^{q} \subset E_{\text {ext }}^{r} \varsubsetneqq E=\operatorname{Rco} E \subset$ Qco $E$.

In Dacorogna-Tanteri [6], it was also proved the existence of the Choquet function for the polyconvex case. The result is the following.

Theorem 34 Let $E \subset \mathbb{R}^{N \times n}$ be a nonempty compact polyconvex set. Then there exists a polyconvex function $\varphi: \mathbb{R}^{N \times n} \rightarrow \mathbb{R} \cup\{+\infty\}$ such that

$$
E_{e x t}^{p}=\{x \in E: \varphi(x)=0\} \quad \text { and } \quad \varphi(x) \leq 0 \Leftrightarrow x \in E .
$$

Acknowledgements: We would like to thank Gisella Croce for interesting discussions on the subject of the article. The research of A. M. Ribeiro was partially supported by portuguese Fundação para a Ciência e Tecnologia (BD/10042/02). 


\section{References}

[1] R. J. Aumann and S. Hart. Bi-convexity and bi-martingales. Israel Journal of Mathematics, 54(2):159-180, 1986.

[2] E. Casadio Tarabusi. An algebraic characterization of quasi-convex functions. Ricerche Mat., 42(1):11-24, 1993.

[3] M. Chlebík and B. Kirchheim. Rigidity for the four gradient problem. J. Reine Angew. Math., 551:1-9, 2002.

[4] B. Dacorogna. Direct methods in the calculus of variations, volume 78 of Applied Mathematical Sciences. Springer-Verlag, Berlin, 1989.

[5] B. Dacorogna and P. Marcellini. Implicit partial differential equations. Progress in Nonlinear Differential Equations and their Applications, 37. Birkhäuser Boston Inc., Boston, 1999.

[6] B. Dacorogna and C. Tanteri. Implicit partial differential equations and the constraints of nonlinear elasticity. J. Math. Pures Appl., 81(4):311-341, 2002.

[7] B. Kirchheim. Deformations with finitely many gradients and stability of quasiconvex hulls. C. R. Acad. Sci. Paris Sér. I Math., 332(3):289-294, 2001.

[8] B. Kirchheim. Rigidity and geometry of microstructures. Preprint MPI for Mathematics in the Sciences, Leipzig, Lecture Note 16, 2003.

[9] J. Kolář. Non-compact lamination convex hulls. Ann. Inst. H. Poincaré Anal. Non Linéaire, 20(3):391-403, 2003.

[10] J. Matoušek and P. Plecháč. On functional separately convex hulls. Discrete Comput. Geom., 19(1):105-130, 1998.

[11] S. Müller. Variational models for microstructure and phase transitions. In Calculus of variations and geometric evolution problems (Cetraro, 1996), volume 1713 of Lecture Notes in Math., pages 85-210. Springer, Berlin, 1999.

[12] S. Müller and V. Šverák. Unexpected solutions of first and second order partial differential equations. In Proceedings of the International Congress of Mathematicians, Vol. II, pages 691-702. Documenta Mathematica, Berlin, 1998.

[13] V. Šverák. On regularity for the Monge-Ampère equation. Preprint HeriotWatt University, 1991.

[14] V. Šverák. New examples of quasiconvex functions. Arch. Rational Mech. Anal., 119(4):293-300, 1992. 
[15] V. Šverák. Rank-one convexity does not imply quasiconvexity. Proc. Roy. Soc. Edinburgh Sect. A, 120(1-2):185-189, 1992.

[16] V. Šverák. On the problem of two wells. In Microstructure and phase transition, volume 54 of IMA Vol. Math. Appl., pages 183-189. Springer, New York, 1993.

[17] L. Tartar. Some remarks on separately convex functions. In Microstructure and phase transition, volume 54 of IMA Vol. Math. Appl., pages 191-204. Springer, New York, 1993.

[18] K. Zhang. On the structure of quasiconvex hulls. Ann. Inst. H. Poincaré Anal. Non Linéaire, 15(6):663-686, 1998.

[19] K. Zhang. On various semiconvex hulls in the calculus of variations. Calc. Var. Partial Differential Equations, 6(2):143-160, 1998.

[20] K. Zhang. Rank 1 connections and the three "well" problem. Preprint. 